\title{
Follow-up of HIV Infected Patients Who Received a Therapeutic Anti-Tat Vaccination Daniel Zagury* ${ }^{*}$, Hélène Le Buanec ${ }^{1}$, Arsene Burny² and Robert C Gallo 3
}

\author{
Address: ${ }^{1}$ Néovacs, Université Pierre et Marie Curie, Paris, France, ${ }^{2}$ Université Libre de Bruxelles, B-1050 Bruxelles, Belgium and ${ }^{3}$ Institute of \\ Human Virology, University of Maryland, Baltimore, MD 21201-1192 \\ Email: Daniel Zagury* - dzagury@neovacs.com \\ * Corresponding author ‡Presenting author
}

from 2005 International Meeting of The Institute of Human Virology

Baltimore, USA, 29 August - 2 September 2005

Published: 8 December 2005

Retrovirology 2005, 2(Suppl I):SII5 doi:I0.II86/I742-4690-2-SI-SII5

Basic and epidemiological documentation as well as non human primate experimentation prompted us to develop anti Tat therapeutic vaccine based on Tat toxoid, a non toxic but immunogenic HIV-1 Tat derivative. Phase I trial conducted at the Hemophiliac Bonomi Center of Milan (Pr. Gringeri) in 1997-1998 and Phase I/II trial organized by Aventis Pasteur showed that the Tat toxoid immunogen adjuvanted with either Seppic oil (ISA51), DcChol or Alum was safe and immunogenic on patients under HAART or not. A structured treatment interruption study (STI) monitored according to EU guidelines was conducted at Brussels (Pr. Clumeck) on the 31 vaccinees who received either a DcChol adjuvanted Tat Toxoid $(\mathrm{n}=12)$, a DcChol placebo $(n=8)$ or non adjuvanted Tat Toxoid $(\mathrm{n}=11)$. Anti-Tat $\mathrm{Ab}$ responders $(\mathrm{n}=9)$ exhibiting both high serum Ab titers $(>10 \mathrm{pg} / \mathrm{ml})$ and a serum anti-Tat neutralizing capacity at the end of the vaccine trial remained significantly HAART-free. By contrast in patients in whom HAART has been prescribed during STI, serum collected prior to treatment did not exercise anti-Tat neutralizing capacity. 